\title{
Strain-rate-dependent deformation behavior in a Ti-based bulk metallic glass composite upon dynamic deformation
}

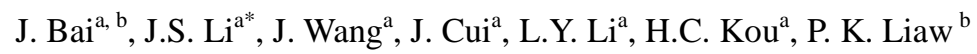 \\ a. State Key Laboratory of Solidification Processing, Northwestern Polytechnical University, Xi'an 710072 ,
}

China

b. Department of Materials Science and Engineering, University of Tennessee, Knoxville, TN 37996-2200, USA

\begin{abstract}
The deformation behavior of in-situ $\mathrm{Ti}_{48} \mathrm{Zr}_{20} \mathrm{Nb}_{12} \mathrm{Cu}_{5} \mathrm{Be}_{15}$ bulk-metallic-glass (BMG) composites was investigated upon dynamic deformation. The present BMG composite exhibits good dynamic mechanical properties, combining high fracture strength $(1850 \mathrm{MPa})$ with remarkable plasticity $(>10 \%)$ at the strain rate of $1.3 \times 10^{3} \mathrm{~s}^{-1}$. Ductile to brittle transition occurs with the increase of strain rates, which can be ascribed to the deteriorated ability of dendrites to impede the propagation of shear bands at higher strain rates. An obvious positive to negative transition on strain rate dependence of flow stresses can be observed with the increasing strain rates. Detailed analysis reveals that the variation from the dendrite-dominated mechanism associated with dislocation movement to the matrix-dominated fracture related to thermal softening is responsible for the present transition. The constitutive equations based on their deformation mechanisms are established for describing the present transition.
\end{abstract}

Keywords: Composite materials; Metallic glasses; Mechanical properties; Strain

\footnotetext{
*Corresponding author. Tel.:+86 298849 1074; fax: +86 2988460294.
}

E-mail address: ljsh@nwpu.edu.cn. 


\section{Introduction}

As a promising candidate of engineering materials, bulk metallic glasses (BMGs) have been extensively explored for their ultra high strength, high hardness and good corrosion resistance [1-3]. However, the poor plasticity accommodated by highly localized shear banding has seriously stymied their application as structural materials. To alleviate the brittleness, synthesizing BMG composites with micron or nano-scale in-situ precipitated crystalline phase during has been rendered as an useful way[4-7]. Especially, in-situ dendrite-toughened BMG composites have attracted great interest due to their distinguished mechanical properties, which share the high strength of glass matrix as well as the large plasticity of dendrites [8-10]. During deformation, these 'soft' second phase with lower shear modulus relative to the glass matrix could effectively homogenize the deformation and stimulate the multiplication of shear bands $[11,12]$. To extend the application of these materials, their deformation behaviors under some extreme conditions, such as high strain rates [13-16], low or high temperature $[7,17,18]$, have been concerned in very recent years.

To date, the strain rate dependent deformation behavior of these in-situ BMG composites has not been clarified yet. The introduction of crystalline dendrites could significantly influence the shear banding behavior in glass matrix $[12,19]$. Singh et al. [20] claimed that the in-situ Zr-based BMG composites exhibited a positive strain rate sensitivity (SRS) that the yield stress increased with the increasing strain rates during quasi-static deformation at or above ambient temperature. Qiao et al. further reported that Ti-based BMG composites still possessed a positive SRS and remarkable dynamic plasticity under dynamic loading, which can be attributed to the effective accommodation of dendrites even upon dynamic loading [14, 21]. However, it was also reported that brittle fracture occurs during dynamic deformation in BMG composites, although large plasticity can be obtained under quasi-static loading[13, 16]. Meanwhile, our previous study 
indicated that a negative SRS could also be observed in BMG composites at cryogenic temperatures upon dynamic deformation [15]. These inconsistent results give an implication that more detailed investigations are still essential to further understand deformation behavior in the in-situ BMG composites.

In the present study, we focus on the strain-rate effects on plastic flow behavior of in-situ Ti-based BMG composites at the ambient temperature. The aim of the present study is to further uncover deformation mechanism in these BMG composites. Moreover, increasing understanding the comprehension of the dynamic deformation behavior of these in-situ BMG composite should aid in extending their application. It is very interesting to find a transition from positive SRS to negative SRS during dynamic deformation in the present BMG composite, which is believed to be associated with the mechanism from the dendrites-dominated plastic deformation to the glass matrix-dominated brittle fracture deformation.

\section{Material and methods}

Ingots of Ti-based BMG composites with a nominal composition of $\mathrm{Ti}_{48} \mathrm{Zr}_{20} \mathrm{Nb}_{12} \mathrm{Cu}_{5} \mathrm{Be}_{15}$ (at. \%) were melted via arc melting the mixture of pure elements ( $\mathrm{Ti}, \mathrm{Zr}, \mathrm{Nb}, \mathrm{Cu}>99.99$ wt. \% and $\mathrm{Be}>99.95$ wt. \%) under a Ti-getterd argon atmosphere. Rod-like samples of $\Phi 3 \mathrm{~mm} \times 50 \mathrm{~mm}$ were obtained by a copper mould casting method. Cylindrical samples with a size of $\Phi 3 \mathrm{~mm} \times 3 \mathrm{~mm}$ were cut and well prepared for the dynamic tests. The dynamic compressive experiments were conducted on a split Hopkinson pressure bar (SHPB) apparatus at the average strain rates from $1.1 \times 10^{3} \mathrm{~s}^{-1}$ to $5.5 \times 10^{3} \mathrm{~s}^{-1}$. The phase composition was examined by the X-ray diffraction with $\mathrm{Cu} K_{a}$ radiation (XRD, DX2700). The as-casted microstructure and deformed samples were observed by the scanning electron microscopy (SEM, VEGA3 TESCAN). High resolution transmission electron microscope (HRTEM, Tecnai G2 F30) was employed to identity the structure evolution.

\section{Results}

The microstructure of the present in-situ BMG composite is presented in Fig .1. Well-developed flower-like 
dendrites (white contrast) with an average span of $15 \mu \mathrm{m}$ are homogenously embedded in the continuous matrix (black contrast). The dendrite volume fraction is estimated to be approximately $52 \%$ by the image analysis. The XRD pattern in Fig. 1(b) further exhibits the crystal peaks of bcc-Ti $(\mathrm{Zr}, \mathrm{Nb})$ solution superimposed on a broad scattering peak, which indicates that the present BMG composite is composed of $\beta$-Ti crystalline dendrites and amorphous matrix.

Fig. 2(a) displays the typical engineering stress vs. strain curves of the present BMG composite upon dynamic deformation at the strain rates from $1.3 \times 10^{3} \mathrm{~s}^{-1}$ to $4.8 \times 10^{3} \mathrm{~s}^{-1}$. It can be seen that the present in-situ BMG composite possesses high flow stress (about 1,850 MPa) as well as remarkable plasticity (>10\%) even upon dynamic deformation at the strain rate of $1.3 \times 10^{3} \mathrm{~s}^{-1}$, implying the potential application under dynamic loading. As strain rate increases to about $2.7 \times 10^{3} \mathrm{~s}^{-1}$, the plasticity reduces to about $9 \%$ and the flow stress rises up to approximately 2,000 MPa. However, brittle fracture dominates the deformation behavior of the present BMG composites when the strain rate is higher than $3.7 \times 10^{3} \mathrm{~s}^{-1}$. Thus, differing from previous studies that plasticity increases with the strain rates under quasi-static loading [22], the ductile to brittle transition occurs in present $\mathrm{BMG}$ composite with the increase of strain rates during dynamic deformation.

The variation of flow stresses with the strain rates was summarized in Fig. 2(b). It can be clearly seen that the flow stress reveals a trend of increasing first and then decreasing with the increasing strain rates, exhibiting a positive to negative SRS transition upon dynamic deformation. This inverted U-shape relationship is notably inconsistent with the monotonous SRS in BMGs or crystalline materials [14, 22-24]. As well known, a positive SRS has been established in crystalline materials, whereas a negative SRS is prevalent in BMGs upon dynamic deformation.

Fig. 3 exhibits the SEM images of the lateral surfaces of the deformed samples at different strain rates. Corresponding to the large plasticity at the strain rate of $1.3 \times 10^{3} \mathrm{~s}^{-1}$, the barreling can be observed in Fig. 3(a). 
The magnified image of lateral surface in Fig. 3(b) further reveals the prevailing of shear bands and slip bands denoted by white and black arrows, respectively, which indicate the effective impediment of dendrites to accommodate the macroscopic deformation of BMG composites and severe plastic deformation inside the dendrites. Fig. 3(c), (e), and (f) are the fractured samples after deformed at $2.7 \times 10^{3} \mathrm{~s}^{-1}, 3.7 \times 10^{3} \mathrm{~s}^{-1}$, and $4.8 \times$ $10^{3} \mathrm{~s}^{-1}$, respectively. All of them show fracture angles less than $45^{\circ}$, which indicate the obvious influence of normal stress during dynamic deformation [25]. Multiple short shear bands and slip bands can also be observed on the magnified lateral surface at $2.7 \times 10^{3} \mathrm{~s}^{-1}$ in Fig. 3(d), in accordance with its plastic flow in Fig. 2(a). However, single shear failure occurs at $3.7 \times 10^{3} \mathrm{~s}^{-1}$ and $4.8 \times 10^{3} \mathrm{~s}^{-1}$, as the corresponding magnified lateral surfaces shown in Fig. 3(f) and (h). Both of them reveal the single shear-band-dominated brittle fracture without any slip bands near the fracture surface.

To further clarify the deformation mechanism of the present BMG composite upon dynamic loading, Fig. 4 shows the microstructure evolution after deformed at $1.3 \times 10^{3} \mathrm{~s}^{-1}$. As displayed in Fig. 4(a), lots of shear bands, marked by black arrows, can be observed in the dendrites, implying the effective accommodation of dendrite to the deformation of glass matrix. The inset a1 is the selected area electronic diffraction (SAED) of dendrites with a zone axis of [-111], which indicates a bcc structure of dendrites with a lattice parameter of a $=0.3372 \mathrm{~nm}$ and no phase transition after deformation. The concentric diffusion rings in inset a2 further demonstrate the good thermal stability of the glass matrix during deformation without nanocrystalization. As marked in Fig. 4(b), amounts of dislocations gather and tangle in dendrites after deformation, which further reveals the severe deformation in the dendrites during plastic deformation. Fig. 4(c) is the HRTEM image of the interface between the dendrites and glass matrix. Atomic bonding between the two phases was confirmed very good. This also ensures the good loading transfer and cooperative deformation between them even upon dynamic deformation. The corresponding inverse fast Fourier transformed (IFFT) images of the rectangular region A and B are 
displayed in Fig. 4(d) and (e). It can be clearly seen that the multiple dislocation denoted by "T" and the lattice distortion marked by arrows are presented in Fig .4(d). Fig. 4(e) demonstrates the maze-like atomic arrangement in the matrix adjacent to the interface, which shows no nanocrystals in the matrix after deformation.

\section{Discussion}

During deformation, the dendrites are considered to exert a crucial role on the propagation of shear bands in BMG composites $[8,16]$. The higher toughness of dendrites is conducive to absorbing the shearing energy and hindering the rapid propagation of shear bands, which results in the formation of more shear bands and slip bands to accommodate the macroscopic plastic deformation [16, 19]. In crystalline materials, it has been indicated that the toughness of materials decreases with the strain rates increase [26, 27]. The higher strain rate leads to the deteriorated toughness of dendrites. That means the shear band will be vulnerable to pass through the dendrite rather than be hindered at higher strain rates. Thus, as shown in Fig. 3, the fracture mode varies from the multiple shear bands and slip bands-coordinated deformation in Fig 3(b) and (d) to single shear-band-dominated brittle fracture in Fig (f) and (h). As a consequence, the plasticity of the present BMG composites decreases along with the increasing strain rates, revealing an obvious ductile to brittle transition during dynamic deformation.

As displayed in Fig 2(b), the present BMG composites possess an obvious positive to negative SRS transition. For convenience, the variation of stresses with strain rates of the present BMG composites is arbitrarily divided to two stages: I. Positive SRS at the relatively low strain rates; II. Negative SRS at the higher strain rates. According to the Taylor dislocation theory [27], the relationship between the critical shear stress of dislocation, $\tau$ and the average velocity, $v$ follows:

$$
v=A \tau^{m}
$$

where, $A$ and $m$ are materials constants. That means higher strain rate requires higher stress to activate 
dislocations movement. Therefore, the crystalline dendrites in the present BMG composites have a positive SRS.

Meanwhile, many previous studies have well documented that a negative SRS dominates the deformation in the glass matrix, especially upon dynamic deformation due to the significant thermal softening [26, 28, 29]. According to the rule of mixture, the positive SRS in stage I demonstrates that the crystalline dendrites instead of the glass matrix assumes more dominance on the deformation [14, 22, 24, 30]. As displayed in Fig. 3 and Fig. 4, severe plastic deformation inside dendrites is characteristic of the formation of abundant slip bands and dislocation, which further indicates the significant accommodation of dendrites to the macroscopic dynamic deformation at relatively low strain rates.

As the strain rate further increases to stage II, the single shear-band-dominated fracture and no slip bands are shown in Fig. 3(f) and (h), which demonstrates that the dendrites with the further deteriorated shear toughness at higher strain rates can be no longer capable to hinder the propagation of a single shear band and little plastic deformation in dendrites. Once a shear band generates in the glass matrix, it cuts through the dendrites straightforward to cause the macroscopic brittleness. Thus, the critical adiabatic shear deformation in the glass matrix plays a dominant role on the strain-rate-dependent deformation behavior. Fig. 5 (a) and (b) exhibit the fracture morphology of the present BMG composites at $3.7 \times 10^{3} \mathrm{~s}^{-1}$ and $4.8 \times 10^{3} \mathrm{~s}^{-1}$, respectively. The fracture surfaces are both covered by melting liquid layers, which reveals the significant temperature rising in the critical shear band. However, compared with that at $3.7 \times 10^{3} \mathrm{~s}^{-1}$, the fracture surface at $4.8 \times 10^{3} \mathrm{~s}^{-1}$ is rougher and more profuse molten traces can also be observed. These obvious differences imply that more temperature rising in the critical shear band at higher strain rates during deformation. Thus, it is reasonable to deduce that more thermal softening further leads to the lower critical shear stress [12, 29]. In other words, the present negative SRS in stage II can be attributed to the thermal-softening effect related to the adiabatic shear deformation in the glass matrix. 
In light of previous work [31], the temperature rising above ambient temperature in the critical shear band at the strain rate of $\dot{\varepsilon}$ can be expressed as:

$$
\Delta T_{\dot{\varepsilon}}=\frac{\delta \chi \tau \dot{\varepsilon} h}{\kappa \cos \theta}
$$

where, $\delta$ is the thickness of critical shear band, about $1 \mu \mathrm{m}[31] \cdot \chi \approx 0.9$ is the fraction of plastic work converted to heat in the critical stress. $\tau \approx \sigma_{\mathrm{yc}} / 2$ is the shear stress and $\sigma_{\mathrm{yc}}$ is the yield stress of BMG composite in stage II. $H=3 \mathrm{~mm}$ is the height of sample. $\theta$ denotes the fracture angles. According to ref [32], $\kappa$ could be taken as 9 $\mathrm{W} /(\mathrm{m} \mathrm{K})$. Then, as shown in Fig. 6, the temperature rising in the critical shear band increases dramatically with the increasing strain rates. Thus, more abundant can be observed at higher strain rates in stage II, which is in accordance with the results in Fig. 5.

As discussed above, the SRS of the present BMG composite is determined by both the dislocation movement induced positive SRS in the dendrites and thermal softening caused negative SRS in the glass matrix. Fig. 7 illustrates the whole strain-rate-dependent deformation behavior of the present BMG composite, together with the crystalline dendrites and the glass matrix. At relatively low strain rate, the positive SRS in the present BMG composite indicates that the dendrites dominate the SRS of the whole composite, whereas, at higher strain rate, the negative SRS implies that the glass matrix plays a dominant role on the SRS of the present composite. Meanwhile, due to the decreased toughness of dendrites with the increase of strain rates, the present BMG composites suffer from ductile to brittle with the increasing strain rates.

Fig. 8 summarizes the variation of stress with strain rate in monolithic BMGs [25, 29, 33-36], BMG composites [14, 20,37, 38]. It can be clearly seen that the BMGs generally exhibit the negative SRS within a wide range of strain rates. Especially, the stresses suffer from a very sharp decrease with the increasing strain rates upon dynamic loading, which has been ascribed to the more dominant thermal softening during dynamic deformation [39]. However, by introducing crystalline second phase, a positive SRS prevails in these BMG 
composites due to the dislocation-related deformation in the reinforced phase [14, 20]. Meanwhile, it should be mentioned that Ta particle-reinforced Zr-based BMG composite also presents a negative SRS [38]. Note that the crystalline volume fraction is only 4\%, dramatically less than the crystalline volumes in the in-situ dendrite-reinforced Ti-, Zr- based BMG composite (> 40\%) [14, 20] or W particle-reinforced Zr-based BMG composite (about $60 \%$ ) [37]. As discussed above, the stage I and stage II depend on the transition from dislocation-dominated mechanism in the crystalline phase to the thermal softening mechanism in the glass matrix. Due to the very low volume fraction of Ta particles, it's reasonable to deduce the dislocation related effects on strain rate sensitivity will be very poor and the thermal softening corresponding to the glass matrix assumes more dominance on strain rate effects within the whole range of strain rates. Thus, these Ta particle-reinforced Zr-based BMG composites are more similar to monolithic BMGs, which exhibit a negative SRS. For comparison, the strain rate dependence of the crystalline composite [40] and $\beta$ titanium alloy[41] are also shown in Fig. 8. Differing from those monolithic BMGs, these crystalline materials possess a positive SRS even at very high strain rates. Therefore, the SRS of the present BMG composite owns the characteristic of both the monolithic BMGs and crystalline materials

To better understand the mechanism of strain-rate-dependent deformation behavior of these in-situ BMG composites, the constitutive equations are established to describe the positive to negative SRS transition. Here, because of the negligible work hardening upon dynamic deformation, the flow stress is taken as the yield stress [14]. As discussed above, the positive SRS of the present BMG composites in stage I, can be attributed to the effect of dendrites related to the dislocation mechanism. For the present BMG composite with a volume fraction of crystalline higher than $50 \%$, the load-bearing model is employed and the flow stress of the present BMG composite can be described as [42],

$$
\sigma_{y c}=\sigma_{y d}\left(1+0.5 f_{m}\right)
$$


here, $\sigma_{\mathrm{yc}}$ and $\sigma_{\mathrm{yd}}$ are the yield stresses of the BMG composite and dendrites, respectively. $f_{\mathrm{m}}=0.48$ is the volume fraction of the glass matrix.

Furthermore, the deformation behavior of the bcc crystalline dendrites can be obtained according to the dislocation-based ZA model by Zerrilli and Armstrong (ZA) [43],

$$
\sigma_{d}=C_{1}+C_{2} \exp \left(-C_{3} T+C_{4} T \ln \dot{\varepsilon}\right)+\mathrm{C}_{5} \varepsilon_{p}^{n}
$$

where, $C_{1}, C_{2}, C_{3}, C_{4}, C_{5}$, and $n$ are materials constants, respectively. $T$ is room temperature. $\dot{\varepsilon}$ is the strain rates. $\varepsilon_{\mathrm{p}}$ is the equivalent plastic strain. At the yielding point, $\varepsilon_{\mathrm{p}}$ is taken as zero. Then, Eq. 3 can be simplified as Eq. 5.

$$
\sigma_{y d}=C_{1}^{\prime}+C_{2}^{\prime} \dot{\varepsilon}^{C_{3}^{\prime}}
$$

here, $C_{1}^{\prime}, C_{2}^{\prime}$ and $C_{3}^{\prime}$ are materials constants.

Substituting Eq. 5 to 3, the relationship between the yield stress of the BMG composite and strain rate can be obtained as Eq. .5 for stage I.

$$
\sigma_{y c}=1.24\left(C_{1}^{\prime}+C_{2}^{\prime} \dot{\varepsilon}^{C_{3}^{\prime}}\right)
$$

On the other hand, as aforementioned, the stage II is dominated by the single shear-band-dominated adiabatic shear mechanism related with the critical shear bands. The critical stress of the glass matrix will capture the flow stress of the present BMG composites. Thus, $\sigma_{\mathrm{yc}}=\sigma_{\mathrm{ym}} / c$ [44]. where, $c \approx 1$ is the average stress concentration factors of the glass matrix [11]. During deformation, it indicates that the formation of critical shear band in the glass matrix can be attributed to the cooperative rearrangement of shear transformation zones (STZs), consisting of a group of atoms and a free volume $[45,46]$ and shear band initiates when the applied stress causes one STZ unstable $[39,47]$.

Considering the thermal softening effect in this stage, according to Liu et al. [39], the temperature rising in STZs with the increase of strain rates upon dynamic deformation are given as 


$$
\Delta T_{S T Z}=\frac{\zeta \Omega \eta \dot{\gamma}^{2}}{8 \pi \kappa r_{0}}
$$

where, $\zeta$ is the correction factor with a value of 3 [47]. $\Omega$ and $\eta$ are STZ volume and the viscosity near STZ, respectively. $\kappa$ denotes the thermal conductivity, and $r_{0}$ is the STZ radius. The shear strain rates, $\dot{\gamma}$, can be calculated by $2 \dot{\varepsilon}$ [39]. Here, $\dot{\varepsilon}$ is the strain rate. According to Refs [32, 39, 47, 48], for the present Ti-based glass matrix, $\eta$, and $r_{0}$ are approximately, $10^{-15} \mathrm{~Pa} \cdot \mathrm{s}$ and $10^{-9} \mathrm{~m}$, respectively, and a medium $4 \mathrm{~nm}^{3}$ is estimated for $\Omega$. Then, $\Delta T_{S T Z} \approx 0.0002 \dot{\varepsilon}^{2}$ is obtained for the present Ti-based glass matrix.

For the macroscopic yielding behavior of the glass matrix, the cooperative shear model (CSM) indicates that the critical strength follows a $t^{2 / 3}$ relation with the temperature of STZ as $[47,49,50]$,

$$
\sigma_{y m}=\sigma_{0}\left(1-C t^{2 / 3}\right)
$$

where, $\sigma_{\mathrm{ym}}$ is the yield stress of the glass matrix at a finite temperature, $T$. As discussed above, $\sigma_{\mathrm{ym}} \approx \sigma_{\mathrm{yc}}$ in stage II for the present BMG composites. $\sigma_{0}$ is the yield stress of the glass matrix at $0 \mathrm{~K}$, obtained by $\sigma_{0}=$ $\sigma_{\mathrm{RT}}\left[1-0.2\left(R T / T_{\mathrm{g}}\right)\right]^{2 / 3}[39]$. Here, the ambient yield stress of the glass matrix, $\sigma_{\mathrm{RT}}$ is about $2 \mathrm{GPa}$ for the present composite. The ambient temperature, $R T$, and the glass transition temperature, $T_{\mathrm{g}}$, are $298 \mathrm{~K}$ and $653 \mathrm{~K}$, respectively. Then, $\sigma_{0}$ is obtained to be $2.5 \mathrm{GPa}$ for the present glass matrix. $t=T / T_{\mathrm{g}}$ and $C$ is a materials constant, irrespective of its negligible correlation with strain rates [50,51].

Then, considering the temperature softening in stage II, the relationship between strain rates and stresses for the present BMG composite can be obtained by combining Eq. 6 with 7 .

$$
\sigma_{y c}=2500\left[1-C\left(\frac{0.0002 \dot{\varepsilon}^{2}+298}{653}\right)^{2 / 3}\right]
$$

Based on Eq. 6 and 9, the fitted results in stage I and stage II are plotted in Fig. 9. Furthermore, according to above discussion, it's reasonable to deduce that the dendrite also dominates the quasi-static SRS deformation behavior of BMG composites. Thus, Eq. 6 can also extrapolate to the quasi-static region. It can be seen that the two models can well describe the variation of stress with the strain rate for the present BMG composite. Then, 
the constitutive equations for the two stages can be obtained to be: $\sigma_{y c}=1,607+0.236 \dot{\varepsilon}^{0.933}$ (stage I) and $\sigma_{y c}=2500\left(1-0.0643\left(\frac{0.0002 \dot{\varepsilon}^{2}+298}{653}\right)^{2 / 3}\right)($ stage II $)$, respectively. Combining them together, the critical strain rate and the maximum stress can be obtained to be about 3,480 s $\mathrm{s}^{-1}$ and 2,080 $\mathrm{MPa}$. That means when the strain rate is lower than about $3,480 \mathrm{~s}^{-1}$, the stress will increase with the increasing strain rates. Otherwise, a negative SRS can be observed at higher strain rates for the present BMG composite. However, more studies are still needed to further uncover the strain-rate-dependent deformation behavior of these BMG composites in the future. For example, the effects of volume fraction, size of dendrites.

\section{Conclusions}

The strain-rate-dependent deformation behavior of in-situ $\mathrm{Ti}_{48} \mathrm{Zr}_{20} \mathrm{Nb}_{12} \mathrm{Cu}_{5} \mathrm{Be}_{15}$ BMG composites were investigated upon dynamic deformation. The present BMG composite shows distinguished plasticity at the strain rates of $1.3 \times 10^{3} \mathrm{~s}^{-1}$. Brittle fracture occurs at high strain rates due to the deteriorated impediment of the dendrites to the propagation of shear bands. The positive to negative strain rate dependent transition was observed with the increasing strain rates upon dynamic deformation, which is believed to be relied on the transition from the dendrite-dominated plastic deformation to shear-band-dominated brittle fracture mechanism.

The constitutive models based on their physical mechanisms are established for the present BMG composite: $\sigma_{y c}=1,607+0.236 \dot{\varepsilon}^{0.933}$ for the positive SRS (stage I) and $\sigma_{y c}=2,500\left[1-0.0643\left(\frac{0.0002 \dot{\varepsilon}+298}{653}\right)^{2 / 3}\right]$ for the negative SRS (Stage II), respectively.

\section{Acknowledgements}

J.S. Li would like to acknowledge the Natural Science Basic Research Plan in Shaanxi Province of China (2014JM6234), Fundamental Research Fund of Northwestern Polytechnical University (JC20120203), Specialized Research Fund for the Doctoral Program of Higher Education (20136102120007) and the Program of Introducing Talents of Discipline to Universities (B08040). P.K. Liaw. appreciates the supports of National 
Science Foundations (DMR-0909037, CMMI-0900271, and CMMI-1100080) and the Department of Energy

(DOE). J. Bai thanks for the fellow ship support of NPU and Chinese Scholarship Council (CSC). 


\section{References}

[1] A. Peker, W. Johnson, Appl. Phys. Lett. 63 (1993) 2342-2344.

[2] Y. Tong, W. Dmowski, Z. Witczak, C.P. Chuang, T. Egami, Acta Mater. 61 (2013) 1204-1209.

[3] J. Wang, W. Cao, L. Wang, S. Zhu, S. Guan, L. Huang, R. Li, T. Zhang, J. Alloys Compd. 637 (2015) 5-9.

[4] C.C. Hays, C.P. Kim, W.L. Johnson, Phys. Rev. Lett. 84 (2000) 2901-2904.

[5] D.C. Hofmann, Science 329 (2010) 1294-1295.

[6] S. Zhu, G. Xie, H. Wang, X. Yang, Z. Cui, A. Inoue, J. Alloys Compd. 586 (2014) 155-158.

[7] P.W.B. Marques, J.M. Chaves, P.S. Silva Jr, O. Florêncio, A. Moreno-Gobbi, L.C.R. Aliaga, W.J. Botta, J. Alloys Compd. 621 (2015) 319-323.

[8] D.C. Hofmann, J.-Y. Suh, A. Wiest, G. Duan, M.-L. Lind, M.D. Demetriou, W.L. Johnson, Nature 451 (2008) 1085-1089.

[9] D.Q. Ma, W.T. Jiao, Y.F. Zhang, B.A. Wang, J. Li, X.Y. Zhang, M.Z. Ma, R.P. Liu, J. Alloys Compd. 624 (2015) 9-16.

[10] J.M. Park, K.R. Lim, E.S. Park, S. Hong, K.H. Park, J. Eckert, D.H. Kim, J. Alloys Compd. 615, Suppl. 1 (2014) S113-S117.

[11] J.W. Qiao, A.C. Sun, E.W. Huang, Y. Zhang, P.K. Liaw, C.P. Chuang, Acta Mater. 59 (2011) 4126-4137.

[12] T.ZHANG, H.Y. He, J.Y. Shi, H.J. Yang, J.W. Qiao, J. Alloys Compd. 583 (2014) 593-597.

[13] Y.S. Wang, G.J. Hao, J.W. Qiao, Y. Zhang, J.P. Lin, Intermetallics 52 (2014) 138-143.

[14] J.W. Qiao, M.Y. Chu, L. Cheng, H.Y. Ye, H.J. Yang, S.G. Ma, Z.H. Wang, Mater. Lett. 119 (2014) 92-95.

[15] J. Bai, H.C. Kou, J. Wang, J.S. Li, R. Hu, Mater. Lett. 117 (2014) 228-230.

[16] J.H. Chen, M.Q. Jiang, Y. Chen, L.H. Dai, Mater. Sci. Eng., A 576 (2013) 134-139.

[17] X.Y. Zhang, Z.Z. Yuan, D.X. Li, J. Alloys Compd. 617 (2014) 670-676. 
[18] J.S. Li, J. Bai, J. Wang, H.C. Kou, R. Hu, H.Z. Fu, Mater. Des. 53 (2014) 737-740.

[19] H.Y. Zhang, G.P. Zheng, J. Alloys Compd. 586, Suppl. 1 (2014) S262-S266.

[20] P.S. Singh, R.L. Narayan, I. Sen, D.C. Hofmann, U. Ramamurty, Mater. Sci. Eng., A 534 (2012) 476-484.

[21] J.W. Qiao, H.Y. Ye, Y.S. Wang, S. Pauly, H.J. Yang, Z.H. Wang, Mater. Sci. Eng., A 585 (2013) 277-280.

[22] J.W. Qiao, Y. Zhang, P. Feng, Q.M. Zhang, G.L. Chen, Mater. Sci. Eng., A 515 (2009) 141-145.

[23] H. Li, G. Subhash, L.J. Kecskes, R.J. Dowding, Mater. Sci. Eng., A 403 (2005) 134-143.

[24] R.L. Narayan, P.S. Singh, D.C. Hofmann, N. Hutchinson, K.M. Flores, U. Ramamurty, Acta Mater. 60 (2012) 5089-5100.

[25] Z.F. Zhang, J. Eckert, L. Schultz, Acta Mater. 51 (2003) 1167-1179.

[26] H.A. Brucka , A.J. Rosakis, W.L. Johnsona, J. Mater. Res. 11 (1996) 503-511.

[27] W.G. Johnston, J.J. Gilman, J. Appl. Phys. 30 (1959) 129-144.

[28] H. Li, G. Subhash, X.-L. Gao, L.J. Kecskes, R.J. Dowding, Scr. Mater. 49 (2003) 1087-1092.

[29] W. Zheng, Y.J. Huang, J. Shen, Mater. Sci. Eng., A 528 (2011) 6855-6859.

[30] J.W. Qiao, P. Feng, Y. Zhang, Q.M. Zhang, G.L. Chen, J. Alloys Compd. 486 (2009) 527-531.

[31] W.D. Liu, K.X. Liu, Intermetallics 19 (2011) 109-112.

[32] G. Wang, Y.J. Huang, D. Makhanlall, J. Shen, J. Mater. Process. Technol. 212 (2012) 1850-1855.

[33] T.C. Hufnagel, T. Jiao, Y. Li, L.Q. Xing, K.T. Ramesh, J. Mater. Res. 17 (2002) 1441-1445.

[34] A.V. Sergueeva, N.A. Mara, D.J. Branagan, A.K. Mukherjee, Scr. Mater. 50 (2004) 1303-1307.

[35] Y.F. Xue, H.N. Cai, L. Wang, F.C. Wang, H.F. Zhang, Mater. Sci. Eng., A 473 (2008) 105-110.

[36] T. Mukai, T.G. Nieh, Y. Kawamura, A. Inoue, K. Higashi, Intermetallics 10 (2002) 1071-1077.

[37] T. Jiao, L.J. Kecskes, T.C. Hufnagel, K.T. Ramesh, Metall. Mater. Trans. A 35 (2004) 3439-3444.

[38] R.T. Ott, F. Sansoz, T. Jiao, D. Warner, J.F. Molinari, K.T. Ramesh, T.C. Hufnagel, C. Fan, Metall. Mater. 
Trans. A 37 (2006) 3251-3258.

[39] W.D. Liu, K.X. Liu, Appl. Phys. Lett. 100 (2012) 141904.

[40] W.-S. Lee, W.-C. Sue, C.-F. Lin, Compos. Sci. Technol. 60 (2000) 1975-1983.

[41] H. Zhan, D. Kent, G. Wang, M.S. Dargusch, Mater. Sci. Eng., A 607 (2014) 417-426.

[42] V.C. Nardone, K.M. Prewo, Scr. Metall. 20 (1986) 43-48.

[43] F.J. Zerilli, R.W. Armstrong, J. Appl. Phys. 61 (1987) 1816-1825.

[44] S.H. Xia, J.T. Wang, Int. J. Plast. 26 (2010) 1442-1460.

[45] A.S. Argon, Acta Metall. 27 (1979) 47-58.

[46] B. Yang, M.L. Morrison, P.K. Liaw, R.A. Buchanan, G. Wang, C.T. Liu, M. Denda, Appl. Phys. Lett. 86 (2005) 141904.

[47] W. Johnson, K. Samwer, Phys. Rev. Lett. 95 (2005) 195501.

[48] S.T. Liu, Z. Wang, H.L. Peng, H.B. Yu, W.H. Wang, Scr. Mater. 67 (2012) 9-12.

[49] T. Dalla, H. Florian, A. Dubach, J. Schällibaum, J.F. Löffler, Acta Mater. 56 (2008) 4635-4646.

[50] Y. Huang, W. Zheng, F. He, J. Shen, Mater. Sci. Eng., A 551 (2012) 100-103.

[51] C. Schuh, T. Hufnagel, U. Ramamurty, Acta Mater. 55 (2007) 4067-4109. 


\section{Figure captions}

Fig. 1 Microstructure (a) and XRD pattern (b) of the present Ti-based BMG composites.

Fig. 2 (a) Typical engineering strain vs. strain curves of the present BMG composites upon dynamic deformation from $1.3 \times 10^{3} \mathrm{~s}^{-1}-4.8 \times 10^{3} \mathrm{~s}^{-1}$. (b) Variation of flow stress with strain rates, indicating the transition from the positive to negative SRS in the present BMG composites.

Fig. 3 Lateral surfaces of fracture samples at different strain rates (a) $1.3 \times 10^{3} \mathrm{~s}^{-1}$, (c) $2.7 \times 10^{3} \mathrm{~s}^{-1}$, (e) $3.7 \times 10^{3}$ $\mathrm{s}^{-1}$, and (g) $4.8 \times 10^{3} \mathrm{~s}^{-1} .(\mathrm{b}),(\mathrm{d}),(\mathrm{f})$, and (h) are the corresponding magnified images of (a), (c), (e), and (g), respectively, demonstrating that the transition from multiple shear bands and slip bands related plastic deformation to single shear bands caused brittle fracture with strain rates increase.

Fig. 4 (a) TEM image of the present BMG composite after deformation at $1.3 \times 10^{3} / \mathrm{s}$, revealing the multiple shear bands propagate in the dendrites. The insets a1 and a2 are the SAED pattern of dendrites with a zone axis of [-111] and the glass matrix, respectively. (b) image of the high density dislocation formed in the dendrites. (c) the HRTEM image of the interface. (d) and (e) the IFFT images of region A and B, showing the multiple dislocation and lattice distortion in the dendrites and maze-like structure in the matrix, respectively.

Fig. 5 Fracture morphology of BMG composites upon dynamic deformation (a) at $3.7 \times 10^{3} \mathrm{~s}^{-1}$ and (b) $4.8 \times 10^{3}$ $\mathrm{s}^{-1}$. More rough and profuse melting layer can be observed on the fracture surface at $4.8 \times 10^{3} \mathrm{~s}^{-1}$, indicating more obvious thermal effect at higher strain rates in stage II.

Fig. 6 Temperature rising in the critical shear band with the increase of strain rates in stage II

Fig. 7 Schematic of the SRS of the crystalline dendrites, glass matrix and BMG composite

Fig. 8 The variation of stress as a function of strain rate in monolithic BMGs, BMG composites, as well as crystalline materials.

Fig. 9 Variation of flow stress within a wide range of strain rates and the corresponding fitting results 
Fig. 1
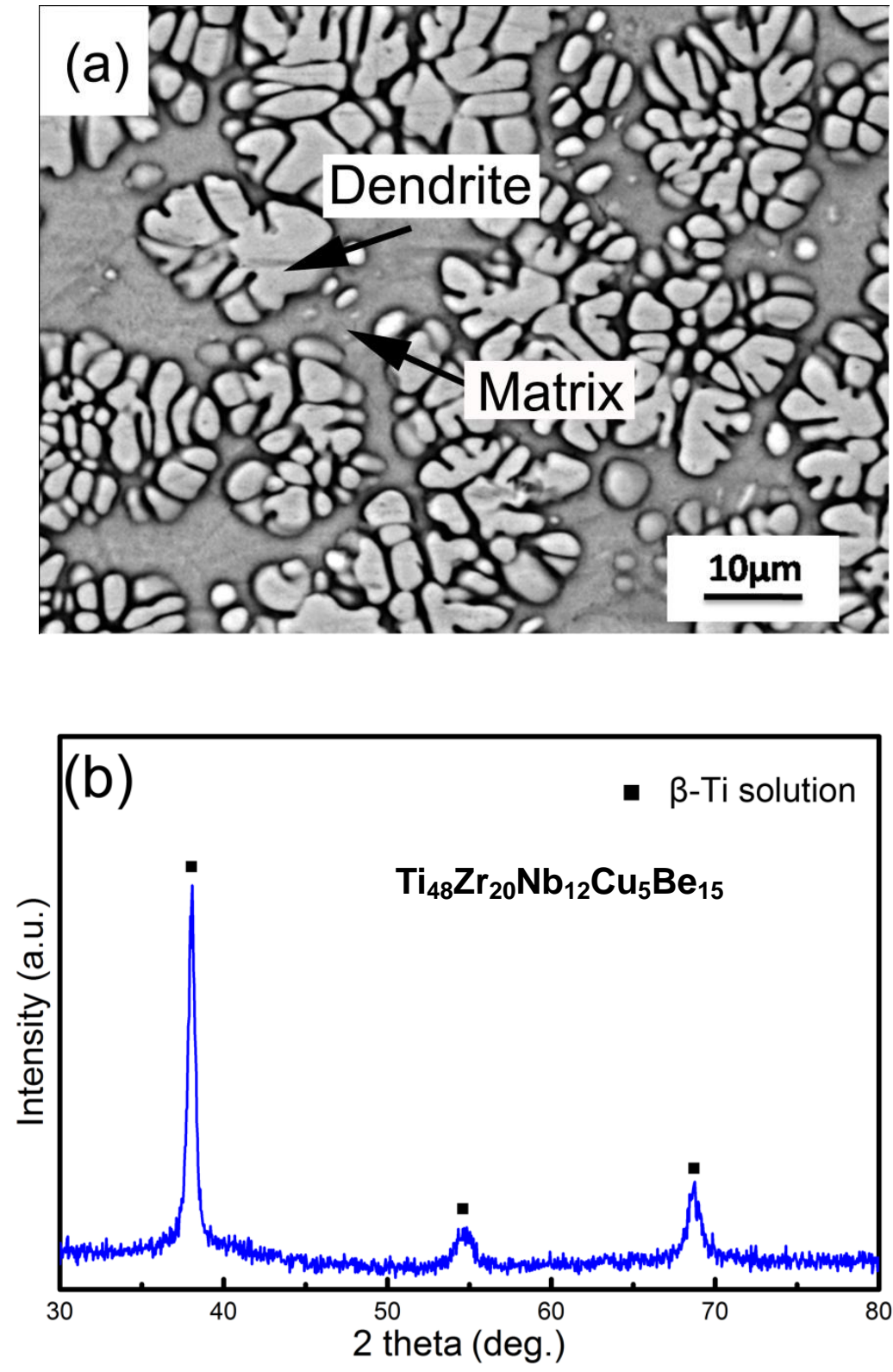

Fig. 1 Microstructure (a) and XRD pattern (b) of $\mathrm{Ti}_{48} \mathrm{Zr}_{20} \mathrm{Nb}_{12} \mathrm{Cu}_{5} \mathrm{Be}_{15}$ BMG composite. 
Fig. 2
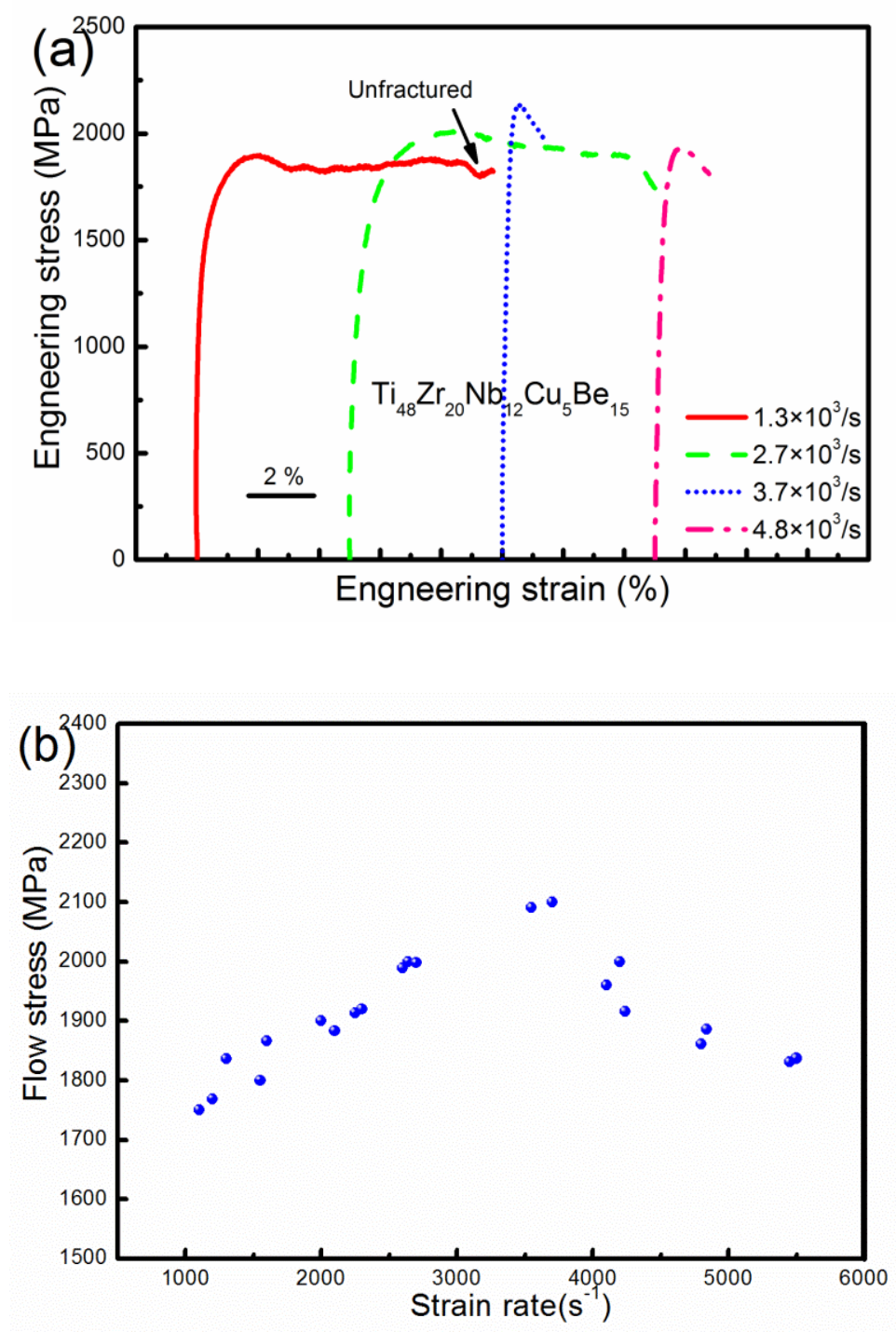

Fig. 2 (a) Typical engineering strain vs. strain curves of $\mathrm{Ti}_{48} \mathrm{Zr}_{20} \mathrm{Nb}_{12} \mathrm{Cu}_{5} \mathrm{Be}_{15} \mathrm{BMG}$ composites upon dynamic deformation from $1.3 \times 10^{3} \mathrm{~s}^{-1}$ to $4.8 \times 10^{3} \mathrm{~s}^{-1}$ and (b) Variation of flow stress with strain rates increase, indicating the transition from positive to negative SRS in the present BMG composites. 
Fig. 3
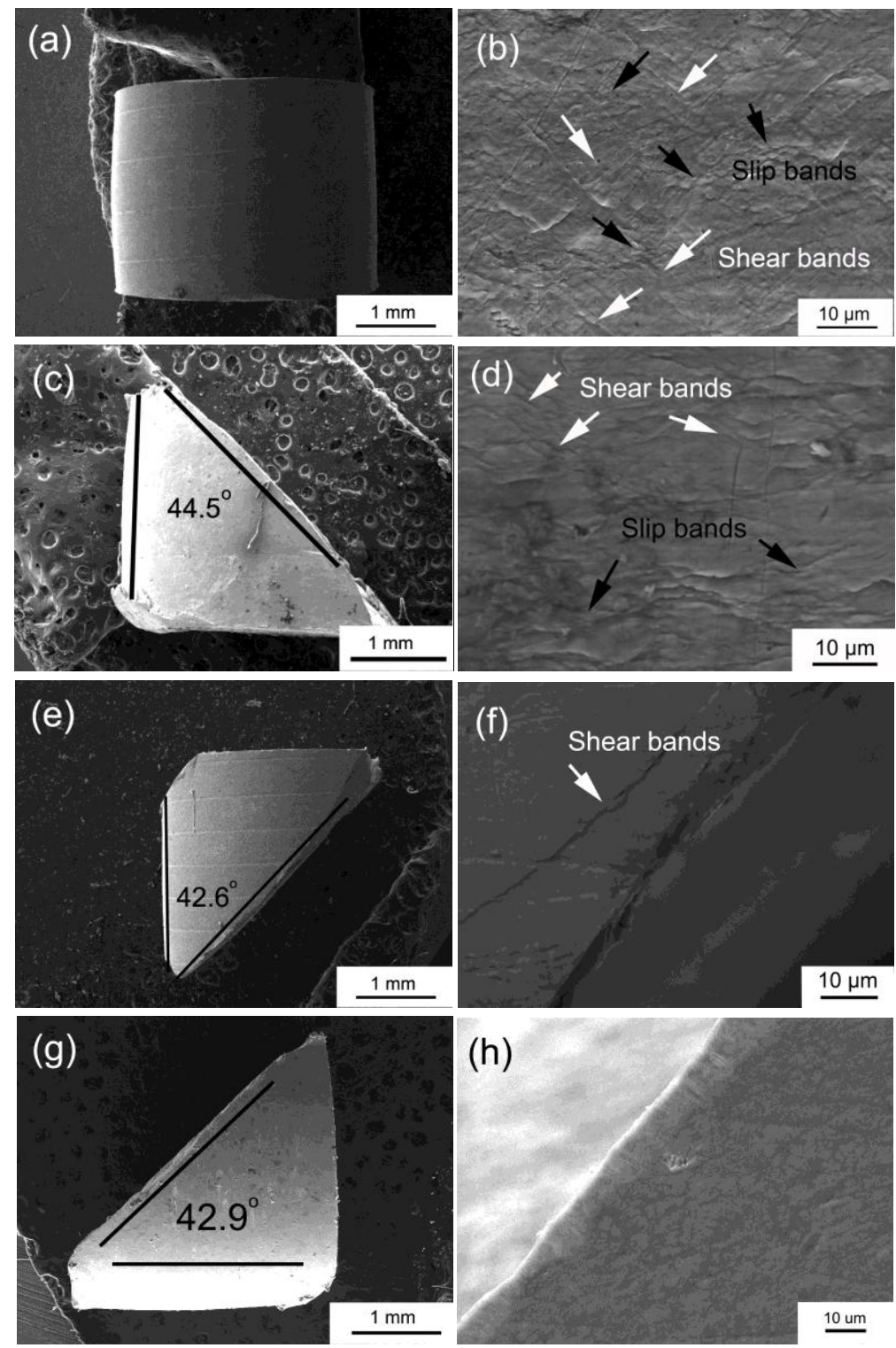

(h)

Fig. 3 Lateral surfaces of fracture samples at different strain rates (a) $1.3 \times 10^{3} \mathrm{~s}^{-1}$, (c) $2.7 \times 10^{3} \mathrm{~s}^{-1}$, (e) $3.7 \times 10^{3}$

$\mathrm{s}^{-1}$, and (g) $4.8 \times 10^{3} \mathrm{~s}^{-1} .(\mathrm{b}),(\mathrm{d}),(\mathrm{f})$, and (h) are the corresponding magnified images of (a), (c), (e), and (g), respectively, demonstrating that the transition from multiple shear bands and slip bands related plastic deformation to single shear bands caused brittle fracture with the strain rate increases. 
Fig. 4
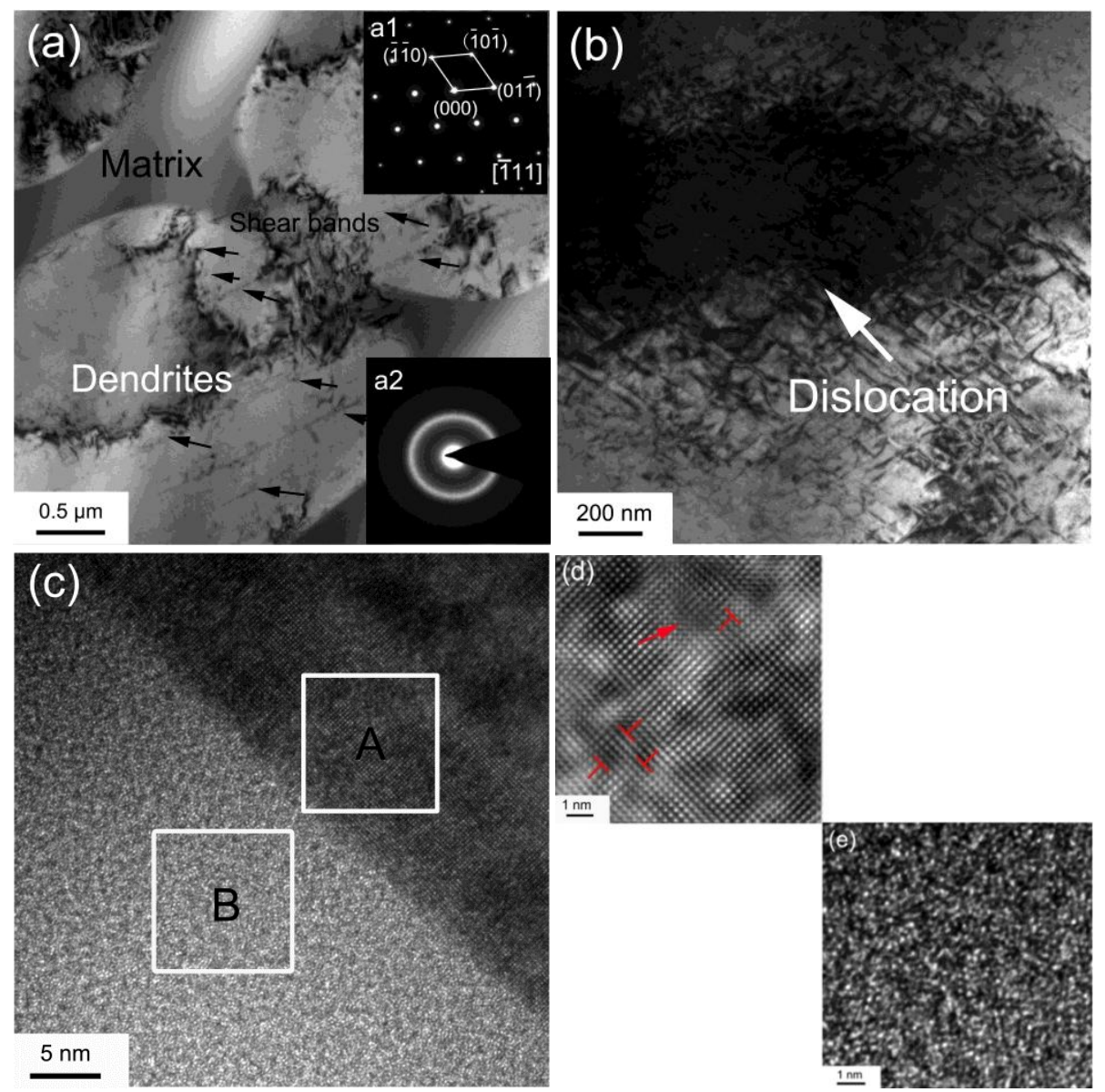

Fig. 4 (a) TEM image of the present BMG composite after deformation at $1.3 \times 10^{3} / \mathrm{s}$, revealing the multiple shear bands propagate in the dendrites. The insets a1 and a2 are the SAED pattern of dendrites with a zone axis of [-111] and the glass matrix, respectively. (b) image of the high density dislocation formed in the dendrites. (c) the HRTEM image of the interface. (d) and (e) the IFFT images of region A and B, showing the multiple dislocation and lattice distortion in the dendrites and maze-like structure in the matrix, respectively. 
Fig. 5
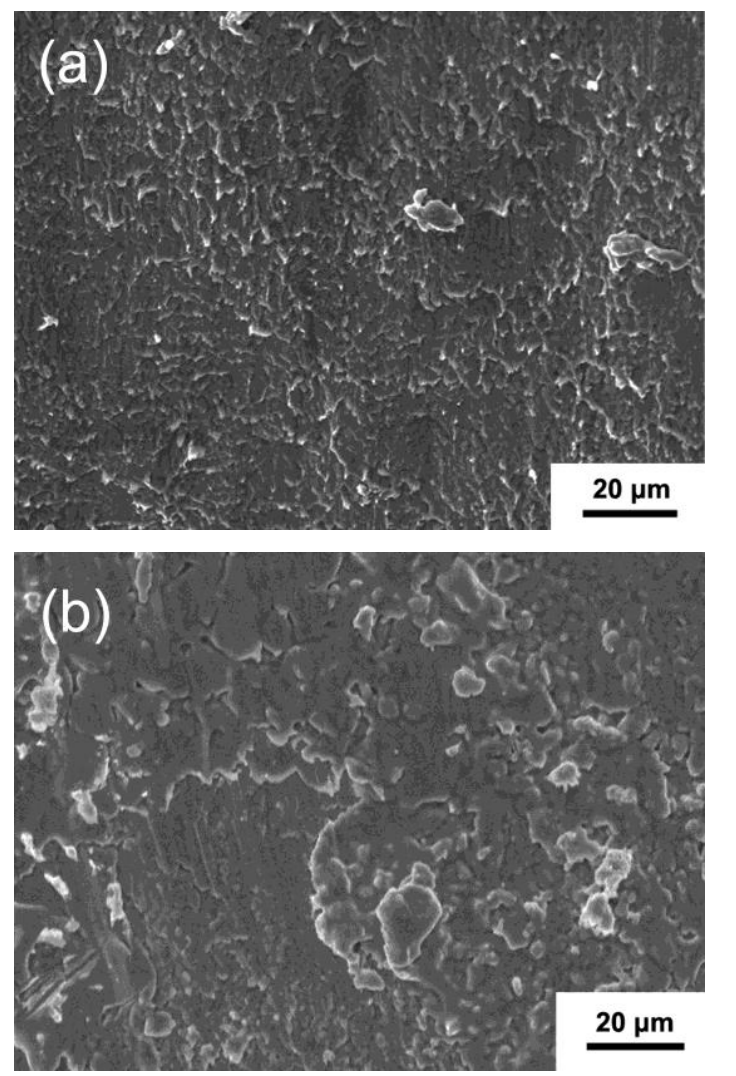

Fig. 5 Fracture morphology of $\mathrm{Ti}_{48} \mathrm{Zr}_{20} \mathrm{Nb}_{12} \mathrm{Cu}_{5} \mathrm{Be}_{15} \mathrm{BMG}$ composites upon dynamic deformation (a) at $3.7 \times 10^{3}$

$\mathrm{s}^{-1}$ and (b) $4.8 \times 10^{3} \mathrm{~s}^{-1}$, respectively. 
Fig. 6

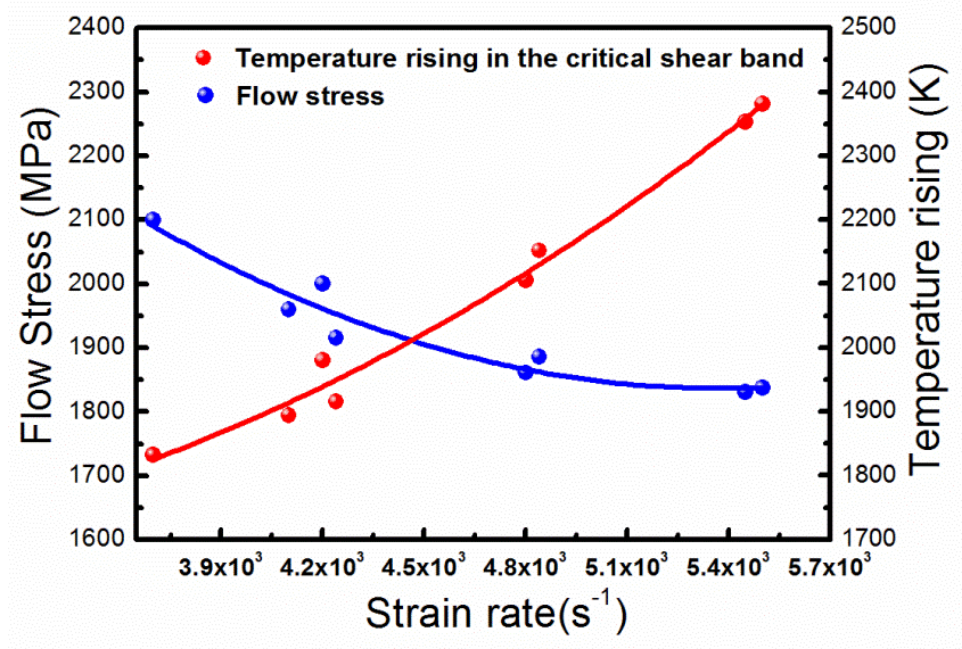

Fig. 6 Temperature rising in the critical shear band with the increase of strain rates in stage II 
Fig. 7

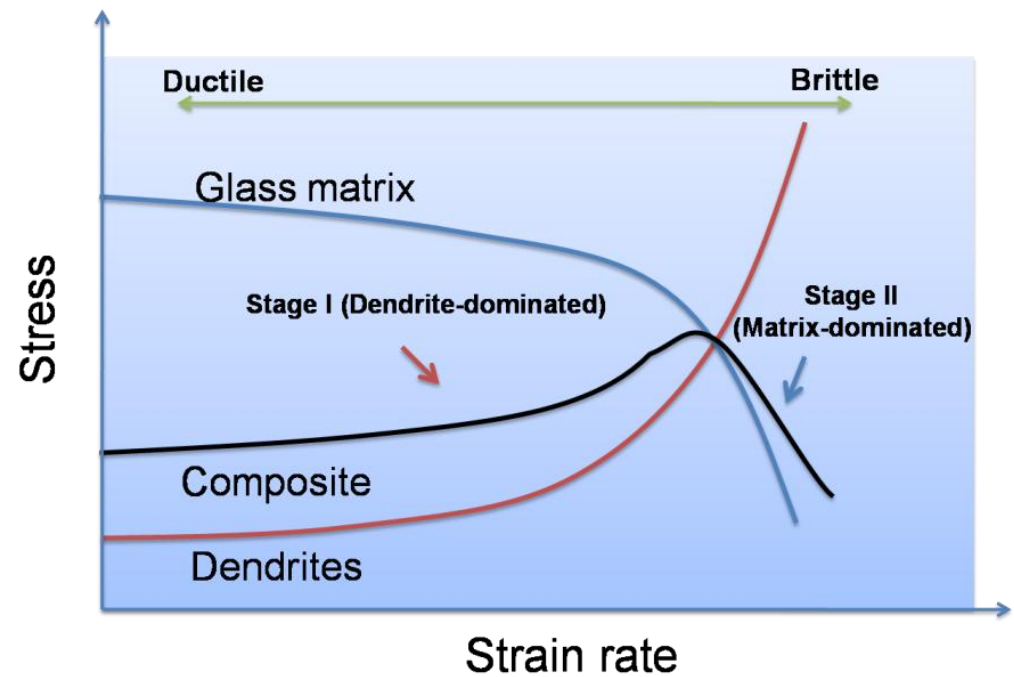

Fig. 7 Schematic of the SRS of the crystalline dendrites, glass matrix, and the present BMG composite 
Fig. 8

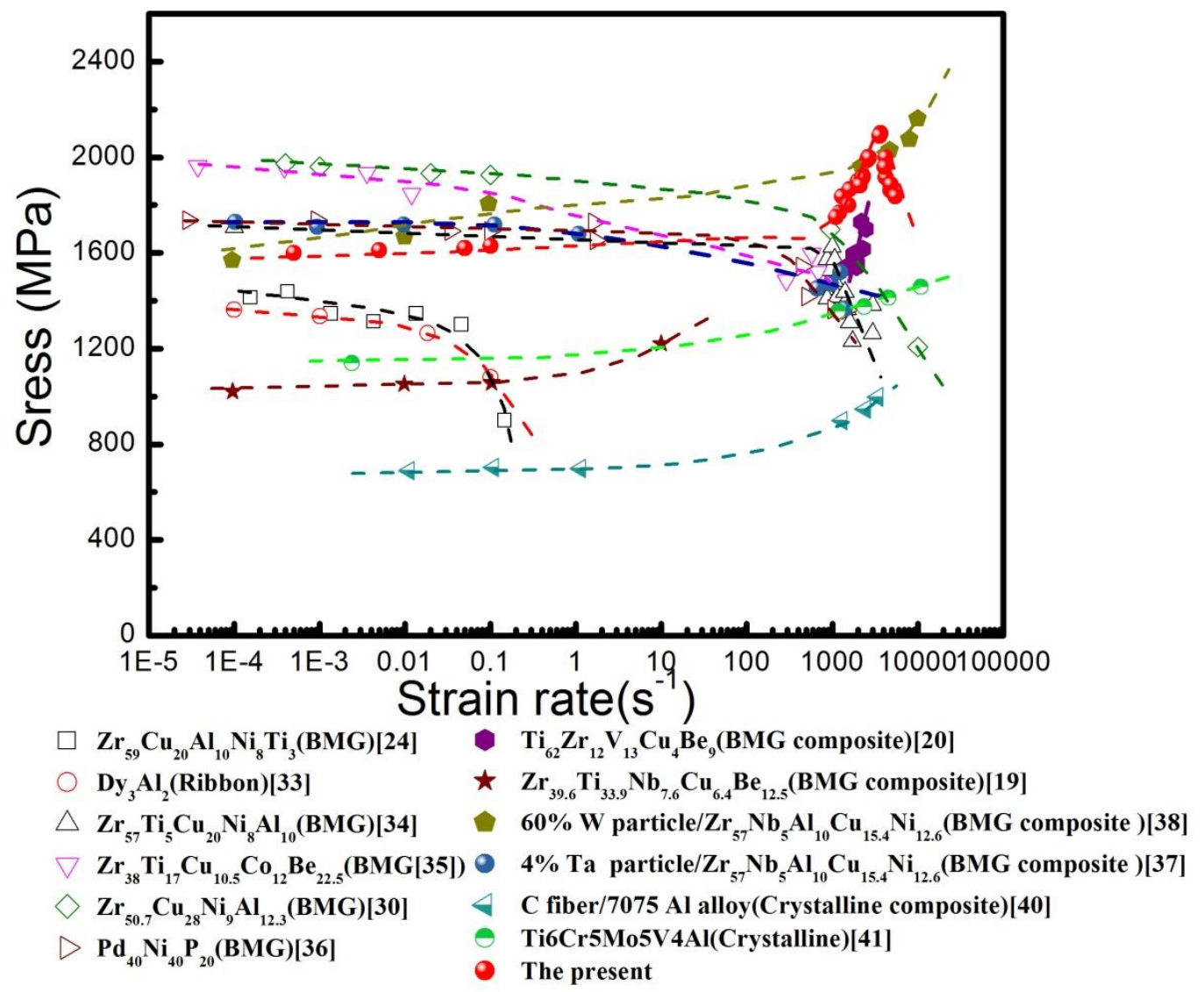

Fig. 8 The variation of stress as a function of strain rate in monolithic BMGs, BMG composites, as well as crystalline materials. 
Fig. 9

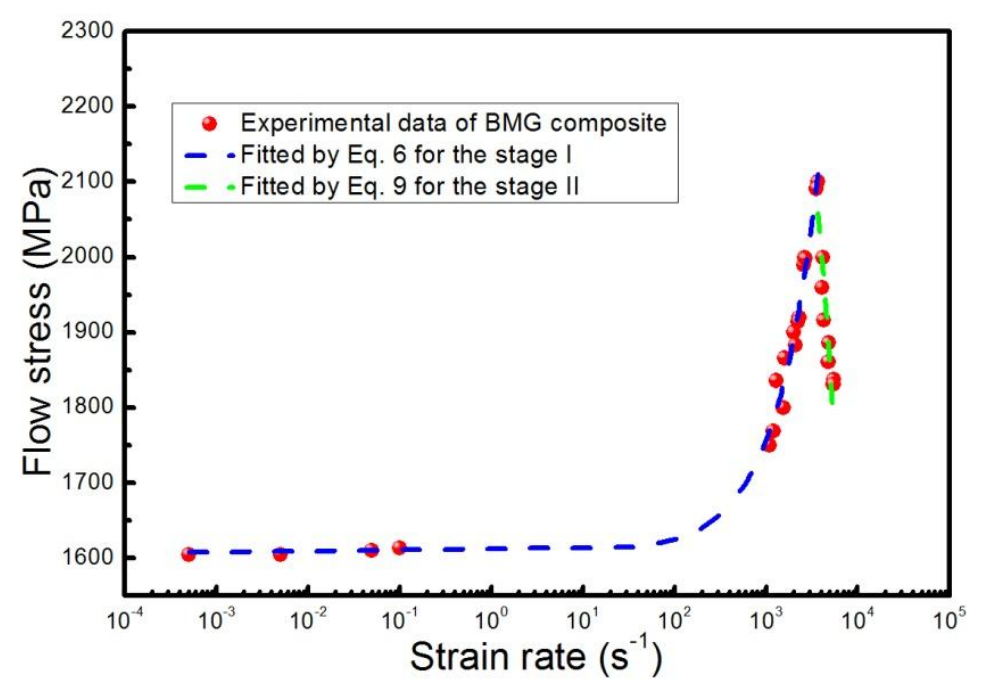

Fig. 9 Variation of flow stress within a wide range of strain rates and the corresponding fitting results 